\title{
A Novel Quantum Inspired Cuckoo Search Algorithm for Bin Packing Problem
}

\author{
Abdesslem Layeb \\ MISC Lab, Computer science department, Mentouri university of Constantine, \\ Constantine, Algeria. \\ Email:layeb@umc.edu.dz \\ Seriel Rayene Boussalia \\ Computer science department, Mentouri University of Constantine, \\ Constantine, Algeria. \\ Email: seriel.rayene@gmail.com
}

\begin{abstract}
The Bin Packing Problem (BPP) is one of the most known combinatorial optimization problems. This problem consists to pack a set of items into a minimum number of bins. There are several variants of this problem; the most basic problem is the onedimensional bin packing problem (1-BPP). In this paper, we present a new approach based on the quantum inspired cuckoo search algorithm to deal with the 1BPP problem. The contribution consists in defining an appropriate quantum representation based on qubit representation to represent bin packing solutions. The second contribution is proposition of a new hybrid quantum measure operation which uses first fit heuristic to pack no filled objects by the standard measure operation. The obtained results are very encouraging and show the feasibility and effectiveness of the proposed approach.
\end{abstract}

Index Terms - Bin Packing Problem, Heuristics, Cuckoo Search Algorithm, Quantum Computing, Hybrid Algorithms

\section{Introduction}

The combinatorial optimization plays a very important role in operational research, discrete mathematics and computer science. The aim of this field is to solve several combinatorial optimization problems that are difficult to solve. Bin packing problem (BPP) is very known NP-Hard optimization problem. There are three main variants of BPP problems: one, two and three dimensional Bin Packing Problems. They have several real applications such as container loading, cutting stock, packaging design and resource allocation, etc. In this paper, we deal with the one-dimensional Bin Packing Problem (1-BPP) [1, 2, 3]. The 1-BPP consists to pack a set of items having different weights into a minimum number of bins which may have also different capacities. Although, this problem seems to be quite simple to define, it has been shown to be NP-hard, because it cannot be solved accurately and optimally in a reasonable time. This is the reasons, why several approximate methods have been proposed to solve this problem, which are generally based on heuristics or metaheuristics. Among the most popular heuristics used to solve the bin packing problem, the First Fit algorithm (FF) which places each item into the first bin in which it will fit. The second popular heuristic algorithm is the Best Fit (BF) which puts each element into the filled bin in which it fits. Moreover, the FF and BF heuristics can be improved by applying a specific order of items like in First Fit Decreasing (FFD) and Best Fit Decreasing (BFD), etc $[4,5,6]$. Moreover, many kinds of metaheuristics have been used to solve the bin packing problems like genetic algorithms [7], Ant colony [8], etc.

Evolutionary computation has been proven to be an effective way to solve complex engineering problems. It presents many interesting features such as adaptation, emergence and learning [9]. Artificial neural networks, genetic algorithms and swarm intelligence are examples of bio-inspired systems used to this end [10]. In recent years, optimizing by swarm intelligence has become a research interest to many research scientists of evolutionary computation fields. There are many algorithms based swarm intelligence like Ant Colony optimization [11, 12], eco-systems optimization [13], etc. The main algorithm for swarm intelligence is Particle Swarm Optimization (PSO) [14, 15], which is inspired by the paradigm of birds grouping. PSO was used successfully in various hard optimization problems. One of the most recent variant of PSO algorithm is Cuckoo Search algorithm (CS). CS is an optimization algorithm developed by Xin-She Yang and Suash Deb in 2009 [16]. It was inspired by the obligate brood parasitism of some cuckoo species by laying their eggs in the nests of other host birds (of other species). Some bird's host can involve direct conflicts with the intruding cuckoos. For example, if a bird's host discovers that the eggs are strange eggs, it will either throw these alien eggs away or simply abandon its nest

and build a new nest elsewhere [17]. The cuckoo's behavior and the mechanism of Lévy flights [18, 19] have leading to design of an efficient inspired algorithm performing optimization search [20, 21]. The recent applications of Cuckoo Search for optimization problems have shown its promising effectiveness. Moreover, a promising discrete cuckoo search algorithm is recently proposed to deal with knapsack 
problems [22].

Quantum Computing (QC) is a new research field that induced intense researches in the last decade, and that covers investigations on quantum computers and quantum algorithms [23]. QC relies on the principles of quantum mechanics like qubit representation and superposition of states. QC is able of processing huge numbers of quantum states simultaneously in parallel. QC brings new philosophy to optimization due to its underlying concepts. Recently, a growing theoretical and practical interest is devoted to researches on merging evolutionary computation and quantum computing [24]. The aim is to get benefit from quantum computing capabilities to enhance both efficiency and speed of classical evolutionary algorithms. This has led to the design of several quantum inspired algorithms such as quantum inspired genetic algorithm [25], quantum differential algorithm [26], quantum inspired scatter search [27], etc. Unlike pure quantum computing, quantum inspired algorithms don't require the presence of a quantum machine to work. Quantum inspired algorithms have been used to solve successfully many combinatorial optimization problems [24, 28]. Recently a new hybrid algorithm called Quantum Inspired Cuckoo Search algorithm (QICSA) is proposed to cope with combinatorial optimization problems [29].The proposed algorithm combines Cuckoo Search algorithm and quantum computing in new one. The features of the proposed algorithm consist in adopting a quantum representation of the search space. The other feature of QICSA is the integration of the quantum operators in the cuckoo search dynamics in order to optimize a defined objective function.

The present study was designed to investigate the use of the QICSA algorithm to deal with the one dimensional bin packing problem. The main features of the proposed approach are the use of the qubit representation to represent the search space and a set of quantum operators operating on this search space. Moreover, we have proposed a new hybrid quantum measure operation based on both the standard quantum measure operation and the First Fist heuristic. We have tested our algorithm on some popular data sets [6] and the results are promising.

The remainder of the paper is organized as follows. In section 2, a formulation of the tackled problem is given. In section 3 , on overview of quantum computing is presented. In section 4 , the cuckoo search algorithm search presented. The proposed method is described in section 5. Experimental results are discussed in section 6. Finally, conclusions and future work are drawn.

\section{Problem formulation}

Bin packing problem is an important task in solving many real problems such as the loading of tractor trailer trucks, cargo airplanes and ships, etc. For example, the container loading problem consists to design an affective loading plan for containers. It consists of finding the most economic storage of articles that have all the same dimensions but with different weights in containers (also called bins) of equal capacity. The constraint is that the bins do not exceed its capacity. This problem can be modeled as a onedimensional bin packing problem. The principal objective is to minimize the number of bins used for storing the set of all items. Formally, the bin packing problem can be stated as follows:

$$
\operatorname{Min} z(y)=\sum_{j=1}^{n} y_{j}
$$

\section{Subject to constraints:}

$$
\begin{array}{ll}
\sum_{j=1}^{n} w_{i} x_{i j} \leq c y_{j} & j \in N^{*+} \\
\sum_{i=1}^{n} x_{i j}=1 & j \in N^{*+} \\
y_{i}, x_{i j} \in\{0,1\} & i, j \in N^{*+}
\end{array}
$$

With:

$y_{i}=1 \quad$ if the bin $i$ is used; else 0

$x_{i j}=1$ if the item $j$ is stocked in bin $i$.

In the above model the objective function is to minimize the total number of bins used to pack all items which have the same capacity ( eq.1). The first constraint guarantees that the weights of items $\left(\mathrm{w}_{\mathrm{i}}\right)$ filled in the bin $\mathrm{j}$ do not exceed the bin capacity. The second constraint ensures that each item is placed only in one bin. It appears to be impossible to obtain exact solutions in polynomial time. The main reason is that the required computation grows exponentially with the size of the problem. Therefore, it is often desirable to find near optimal solutions to these problems. Efficient heuristic algorithms offer a good alternative to accomplish this goal. Within this perspective, we are interested in applying a QICSA algorithm.

\section{Overview of Quantum Computing}

Quantum computing is a new theory which has emerged as a result of merging computer science and quantum mechanics. Its main goal is to investigate all the possibilities a computer could have if it followed the laws of quantum mechanics. The origin of quantum computing goes back to the early 80 when Richard Feynman observed that some quantum mechanical During the last decade, quantum computing has attracted widespread interest and has induced intensive investigations and researches since it appears more powerful than its classical counterpart. Indeed, the parallelism that the quantum computing provides reduces obviously the algorithmic complexity. Such an 
ability of parallel processing can be used to solve combinatorial optimization problems which require the exploration of large solutions spaces. The basic definitions and laws of quantum information theory are beyond the scope of this paper. For in-depth theoretical insights, one can refer to [23].

The qubit is the smallest unit of information stored in a two-state quantum computer. Contrary to classical bit which has two possible values, either 0 or 1 , a qubit will be in the superposition of those two values. The state of a qubit can be represented by using the bracket notation:

$$
|\Psi\rangle=\alpha|0\rangle+\beta|1\rangle
$$

where $|\Psi\rangle$ denotes more than a vector $\vec{\Psi}$ in some vector space. $|0\rangle$ and $|1\rangle$ represent the classical bit values 0 and 1 respectively; $a$ and $b$ are complex numbers such that:

$|a|^{2}+|b|^{2}=1$

$a$ and $b$ are complex number that specify the probability amplitudes of the corresponding states. When we measure the qubit's state we may have ' 0 ' with a probability $|a|^{2}$ and we may have ' 1 ' with a probability $|b|^{2}$. A system of m-qubits can represent $2^{\mathrm{m}}$ states at the same time. Quantum computers can perform computations on all these values at the same time. It is this exponential growth of the state space with the number of particles that suggests exponential speed-up of computation on quantum computers over classical computers. Each quantum operation will deal with all the states present within the superposition in parallel. When observing a quantum state, it collapses to a single state among those states.

Quantum Algorithms consist in applying successively a series of quantum operations on a quantum system. Quantum operations are performed using quantum gates and quantum circuits. It should be noted that designing quantum algorithms is not easy at all. Yet, there is not a powerful quantum machine able to execute the developed quantum algorithms. Therefore, some researchers have tried to adapt some properties of quantum computing in the classical algorithms. Since the late 1990s, merging quantum computation and evolutionary computation has been proven to be a productive issue when probing complex problems. Like any other EA, a Quantum Evolutionary Algorithm (QEA) relies on the representation of the individual, the evaluation function and the population dynamics. The particularity of QEA stems from the quantum representation they adopt which allows representing the superposition of all potential solutions for a given problem. It also stems from the quantum operators it uses to evolve the entire population through generations. QEA has been successfully applied on many problems [24, 28, 29].

\section{Cuckoo Search Algorithm}

In order to solve complex problems, ideas gleaned from natural mechanisms have been exploited to develop heuristics. Nature inspired optimization algorithms has been extensively investigated during the last decade paving the way for new computing paradigms such as neural networks, evolutionary computing, swarm optimization, etc. The ultimate goal is to develop systems that have ability to learn incrementally, to be adaptable to their environment and to be tolerant to noise. One of the recent developed bioinspired algorithms is the Cuckoo Search (CS) [16] which is based on style life of Cuckoo bird. Cuckoos use an aggressive strategy of reproduction that involves the female hack nests of other birds to lay their eggs fertilized. Sometimes, the egg of cuckoo in the nest is discovered and the hacked birds discard or abandon the nest and start their own brood elsewhere. The Cuckoo Search proposed by Yang and Deb 2009 [16] is based on the following three idealized rules:

- Each cuckoo lays one egg at a time, and dumps it in a randomly chosen nest;

- The best nests with high quality of eggs (solutions) will carry over to the next generations;

- The number of available host nests is fixed, and a host can discover an alien egg with a probability pa $\in[0,1]$. In this case, the host bird can either throw the egg away or abandon the nest so as to build a completely new nest in a new location.

The last assumption can be approximated by a fraction pa of the $\mathrm{n}$ nests being replaced by new nests (with new random solutions at new locations). The generation of new solutions $x(t+1)$ is done by using a Lévy flight (eq.7). Lévy flights essentially provide a random walk while their random steps are drawn from a Lévy distribution for large steps which has an infinite variance with an infinite mean (eq.8). Here the consecutive jumps/steps of a cuckoo essentially form a random walk process which obeys a power-law steplength distribution with a heavy tail [16].

$$
\begin{aligned}
& x_{i}^{l+1}=x_{i}^{l}+\alpha \oplus \operatorname{Lévy}(\lambda) \\
& L \grave{e} v y \sim \mathrm{u}=\mathrm{t}^{-\wedge}
\end{aligned}
$$

where $\alpha>0$ is the step size which should be related to the scales of the problem of interest. Generally we take $\alpha=\mathrm{O}(1)$. The product $\oplus$ means entry-wise multiplications. This entry-wise product is similar to those used in PSO, but here the random walk via Lévy flight is more efficient in exploring the search space as its step length is much longer in the long run. 
The main characteristics of CS algorithm it's its simplicity. In fact, comparing with other population or agent-based metaheuristic algorithms such as particle swarm optimization and harmony search, there are few parameters to set. The applications of CS into engineering optimization problems have shown its encouraging efficiency. For example, a promising discrete cuckoo search algorithm is recently proposed to solve nurse scheduling problem [19]. Another binary version of cuckoo search is proposed in [22] to sole the knapsack problems. An efficient computation approach based on cuckoo search has been proposed for data fusion in wireless sensor networks [20]. In more details, the proposed cuckoo search algorithm can be described as follow:

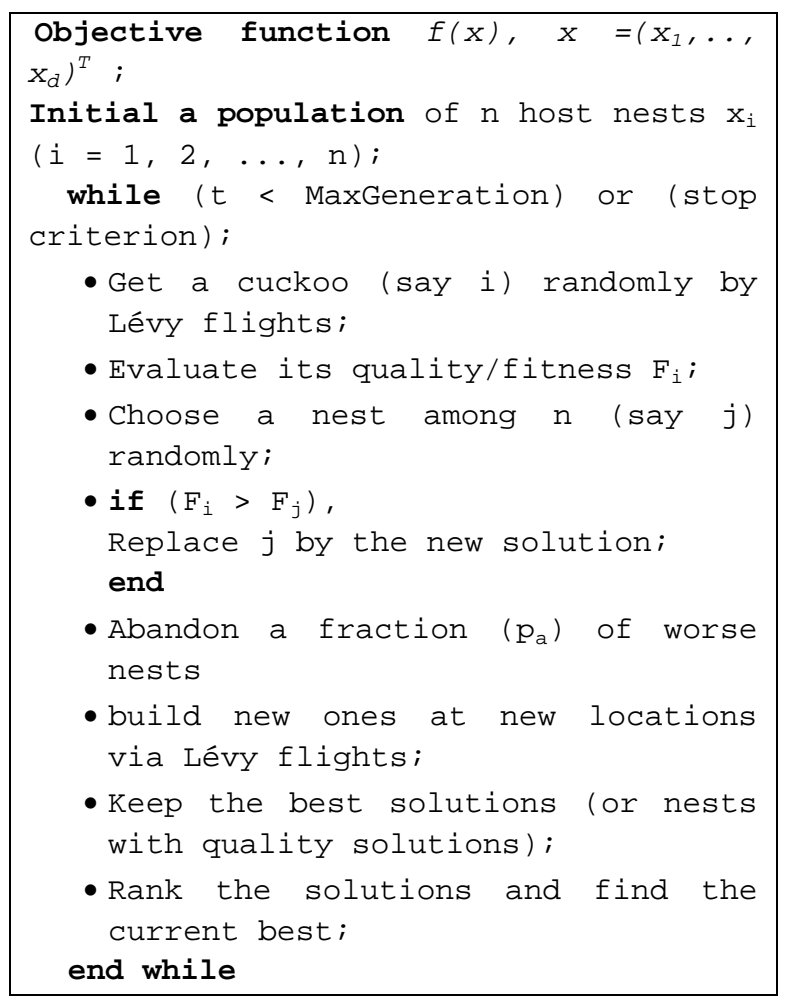

Fig. 1. Cuckoo Search Schema.

\subsection{Quantum Inspired Cuckoo Search}

In section, we present the Quantum Inspired Cuckoo Search (QICSA) which integers the quantum computing principles such as qubit representation, measure operation and quantum mutation, in the core the cuckoo search algorithm. This proposed model will focus on enhancing diversity and the performance of the cuckoo search algorithm [29].

The QICSA architecture, which has been developed to solve combinatorial problems, is explained in the Figure 2. Our architecture contains three essential modules. The first module contains a quantum representation of cuckoo swarm. The particularity of quantum inspired cuckoo search algorithm stems from the quantum representation it adopts which allows representing the superposition of all potential solutions for a given problem. Moreover, the generation of a new cuckoo depends on the probability amplitudes $a$ and $b$ of the qubit function $\Psi$ (eq.5). The second module contains the objective function and the selection operator. The selection operator is similar to the elitism strategy used in genetic algorithms. Finally, the third module, which is the most important, contains the main quantum cuckoo dynamics. This module is composed of 4 main operations inspired from quantum computing and cuckoo search algorithm: Measurement, Mutation, Interference, and Lévy flights operations. QICSA uses these operations to evolve the entire swarm through generations [29].

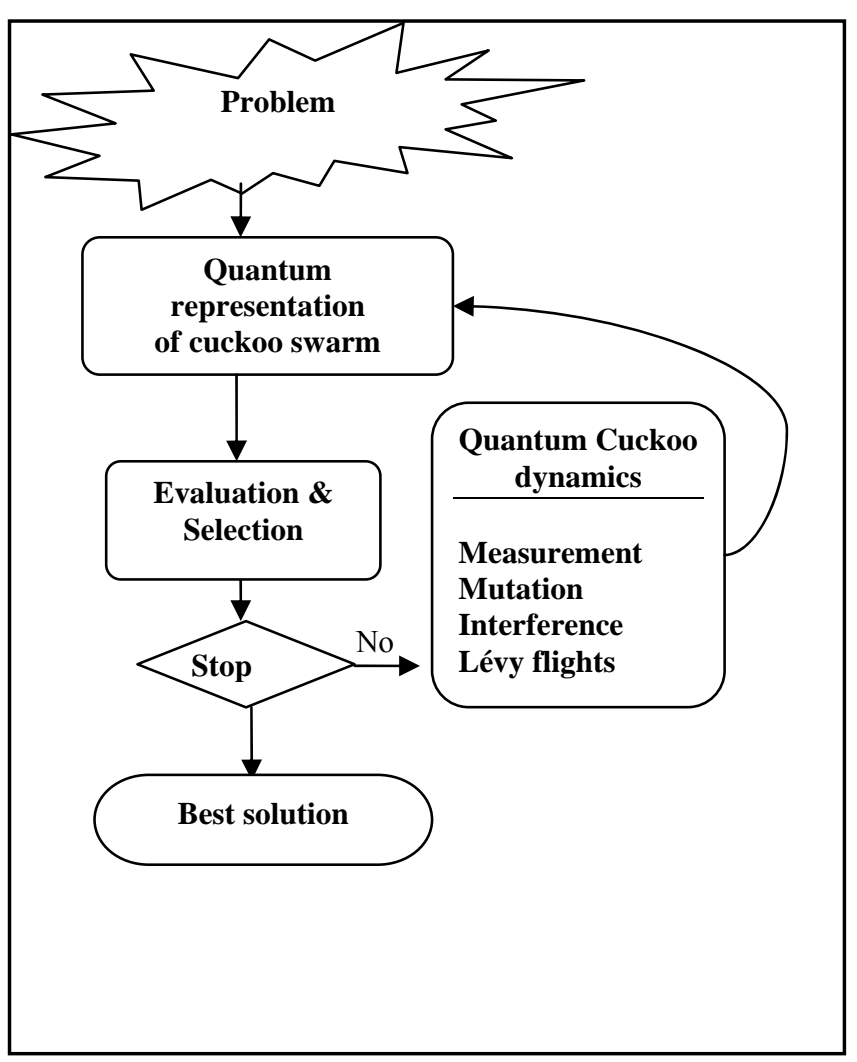

Fig. 2. Architecture of the QICSA algorithm [29]

\section{The proposed approach for solving the bin packing problem}

The development of the suggested approach called QICSABP is based mainly on a quantum representation of the searchspace associated with the problem and a QICSA dynamic used to explore this space by operating on the quantum representation by using quantum operations. In order to show how quantum computing concepts have been tailored to the problem at hand, we need first to derive a representation scheme which includes the definition of an appropriate quantum representation of potential pin packing solutions and the definition of quantum operators. Then, we describe how these defined concepts have been integrated in cuckoo search algorithm 


\subsection{Quantum representation of variable order}

In order to easily apply quantum principles on bin packing problem, we need to map potential solutions into a quantum representation that could be easily manipulated by quantum operators. The bin packing solution is represented as binary matrix (Figure.3) satisfying the following criteria:

For $\mathrm{N}$ objects, the size of the binary matrix is $N^{*} N$. The columns represent the bins and the rows represent the objects.

$>$ The presence of 1 in the position $(i, j)$ indicates that the object $i$ is filled in the bin $i$

$>$ In each row there is a single 1, i.e. the object is filled in one bin.

The following example shows a binary solution for bin packing instance of 4 objects. According to the example the objects 2 and 3 are packed in the bin 2, the object 2 is filled in the bin 1 , and the object 4 is filled in the bin 3 . So the number of bins used is 3 , the last bin is not used.

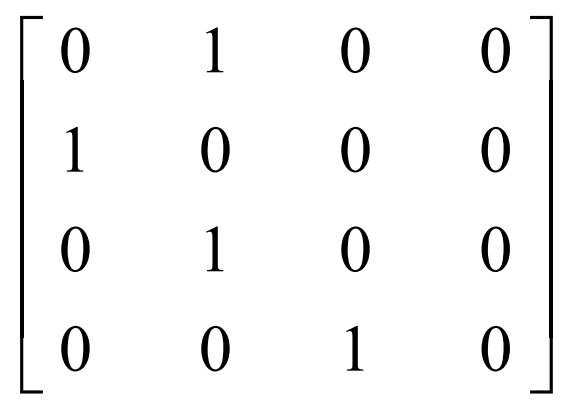

Fig. 3. Binary representation of bin packing solution.

In terms of quantum computing, each variable emplacement is represented as a quantum register as shown in Figure 4. One quantum register contains a superposition of all possible variable positions. Each column $\left(\begin{array}{l}a_{i} \\ b_{i}\end{array}\right)$ represents a single qubit and corresponds to the binary digit 1 or 0 . The probability amplitudes $a_{i}$ and $b_{i}$ are real values satisfying $\left|a_{i}\right|^{2}+\left|b_{i}\right|^{2}=1$. For each qubit, a binary value is computed according to its probabilities $\left|a_{i}\right|^{2},\left|b_{i}\right|^{2}$, and the bin capacity. $\left|a_{i}\right|^{2}$ and $\left|b_{i}\right|^{2}$ can be interpreted as the probabilities to have respectively 0 or 1 . Consequently, all feasible variable orders can be represented by a quantum matrix $Q M$ (Figure 5) that contains the superposition of all possible variable permutations. This quantum matrix can be viewed as a probabilistic representation of all potential bin packing solutions. When embedded within a cuckoo search algorithm framework, it plays the role of a nest. A quantum representation offers a powerful way to represent the solution space and reduces consequently the required number of cuckoo. Only one quantum matrix is needed to represent the entire swarm.

$$
\left(\begin{array}{l|ll|ll}
\mathbf{a}_{1} & \mathbf{a}_{2} & & \mathbf{a}_{\mathbf{m}} \\
\mathbf{b}_{1} & \mathbf{b}_{2} & & \mathbf{b}_{\mathbf{m}}
\end{array}\right)
$$

Fig.4. Quantum register encoding a row in the binary matrix.

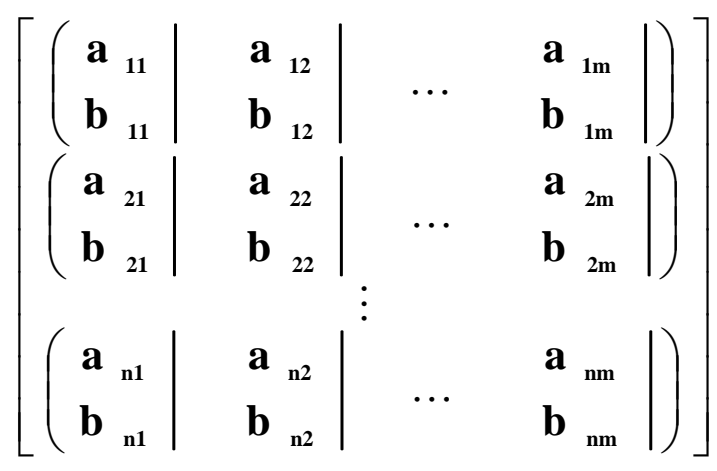

Fig. 5. Quantum representation of variable ordering.

\subsection{Quantum operators}

The quantum inspired cuckoo search algorithm uses some of quantum inspired operations like measurement, interference, and mutation. This integration helps to increase the optimization capacities of the cuckoo search.

\subsubsection{Measurement}

This operation transforms by projection the quantum vector into a binary vector (figure 6). Therefore, there will be a solution among all the solutions present in the superposition. But contrary to the pure quantum theory, this measurement does not destroy the superposition. That has the advantage of preserving the superposition for the following iterations knowing that we operate on traditional machines. The binary values for a qubit are computed according to its probabilities $\left|a_{i}\right|^{2}$ and $\left|b_{i}\right|^{2}$.

For the bin packing problem, this operation is accomplished as follows: for each qubit, we generate a random number $\operatorname{Pr}$ between 0 and 1 ; the value of the corresponding bit is 1 if the value $\left|b_{i}\right|^{2}$ is greater than $P r$, and otherwise the bit value is 0 . However, the use the standard measure operation defined in [29] can lead to infeasible solutions and then increase the computational time of the algorithm to find good solutions. By using the standard measure, we can get an overloaded bin or unpacked item (we can get a zero vector for variable emplacement). In order to delete this kind of solutions, we have introduced bin capacity in the measure operation. So, the value 1 is obtained if the 
value $\left|b_{i}\right|^{2}$ is greater than the random number $\operatorname{Pr}$, and the item weight is less than the bin capacity. To correct the unpacked item, we have introduced the FF in the core the measure operation heuristic to pack the unfilled object. The FF algorithm is used if we have a zero vector for a given object.

It should be noted that, the measurement operation can be seen also as a diversification operator. Indeed, two successive measurements do not give necessarily the same solution which increases the diversification capacities of our approach. The obtained binary vector is then translated into the problem solution. Consequently, with one quantum Matrix, we can get several different solutions for the bin packing problem, which gives a great diversity to the cuckoo search algorithm.

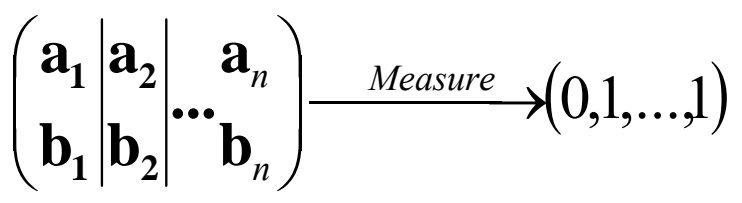

Fig.6. Quantum measurement.

\subsubsection{Quantum interference}

This operation amplifies the amplitude of the best solution and decreases the amplitudes of the bad ones. It primarily consists in moving the state of each qubit in the direction of the corresponding bit value in the best solution in progress. The operation of interference is useful to intensify research around the best solution and it plays the role of local search method. This operation can be accomplished by using a unit transformation which achieves a rotation whose angle is a function of the amplitudes $a_{i}, b_{i}$ (figure 7). The rotation angle's value $\delta \theta$ should be well set in order avoid premature convergence. A big value of the rotation angle can lead to premature convergence or divergence; however a small value to this parameter can increase the convergence time. Consequently, the angle is set experimentally and its direction is determined as a function of the values of $a_{i}, b_{i}$ and the corresponding element's value in the binary vector (table 1$)$. In our algorithm, we have set the rotation angle $\delta \theta=\mathrm{pi} / 20$. However, we can use a dynamic value of the rotation angle in order to increase the performance of the interference operation [29].

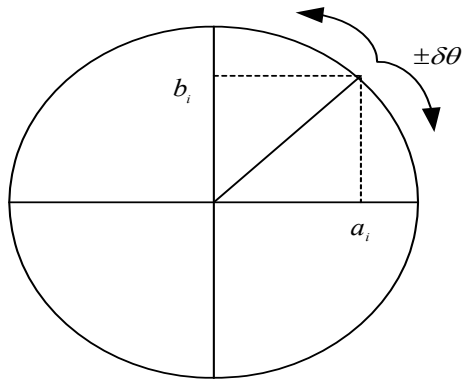

Fig. 7. Quantum interference.

Table 1. Lookup table of the rotation angle

\begin{tabular}{|l|l|l|l|}
\hline $\mathrm{a}$ & $\mathrm{b}$ & $\begin{array}{c}\text { Reference bit } \\
\text { value }\end{array}$ & Angle \\
\hline$>0$ & $>0$ & 1 & $+\delta \theta$ \\
\hline$>0$ & $>0$ & 0 & $-\delta \theta$ \\
\hline$>0$ & $<0$ & 1 & $-\delta \theta$ \\
\hline$>0$ & $<0$ & 0 & $+\delta \theta$ \\
\hline$<0$ & $>0$ & 1 & $-\delta \theta$ \\
\hline$<0$ & $>0$ & 0 & $+\delta \theta$ \\
\hline$<0$ & $<0$ & 1 & $+\delta \theta$ \\
\hline$<0$ & $<0$ & 0 & $-\delta \theta$ \\
\hline
\end{tabular}

\subsubsection{Mutation operator}

This operator is inspired from the evolutionary mutation. It allows moving from the current solution to one of its neighbours. This operator allows exploring new solutions and thus enhances the diversification capabilities of the search process. In each generation, the mutation is applied with some probability. We distinguish two types of quantum mutations:

- Inter-qubit Mutation: this operator performs permutation between two qubits. It consists first in selecting randomly a register in the quantum matrix. Then, pairs of qubits are chosen randomly according to a defined probability (figure 8).

- Intra-qubit Mutation: it consists in selecting randomly a qubit according to a defined probability, next we make a permutation between the qubit amplitudes $a_{i}$ et $b_{i}$ as it is shown on Figure 9.

View that the quantum inspired cuckoo search characteristics offers a great diversity; it is recommended to use small values for the of mutation probability in order to keep good performance of the quantum inspired cuckoo search. 


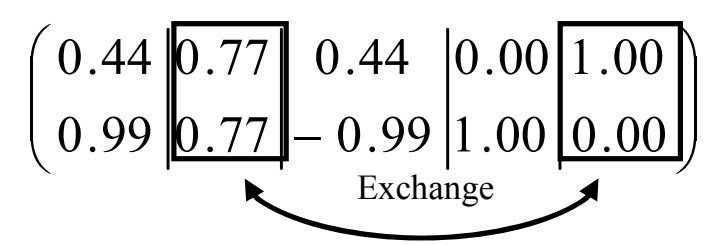

Fig. 8. inter-qubit quantum mutation.

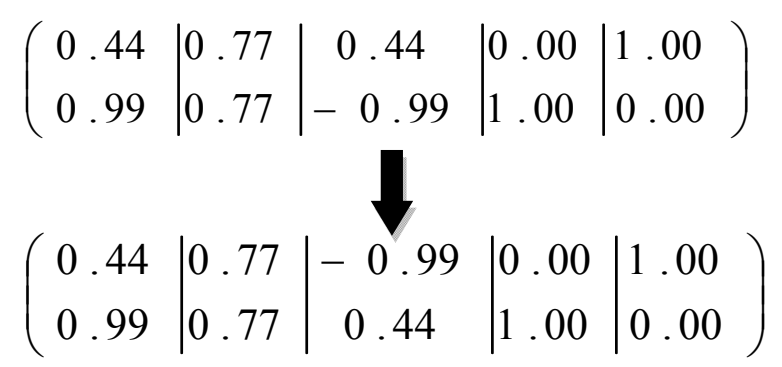

Fig. 9. intra-qubit quantum mutation.

\subsection{Outlines of the proposed algorithm}

Now, we describe how the representation scheme including quantum representation and quantum operators has been embedded within a cuckoo search algorithm and resulted in a hybrid stochastic algorithm used to solve the bin packing problem. Firstly, a swarm of $p$ host nest is created at random positions to represent all possible solutions. The developed algorithm called QICSABP progresses through a number of generations according to the QICSA dynamics. During each iteration, the following main tasks are performed. A new cuckoo is built using the Lévy flights operator followed by the quantum mutation which is applied with some probability $p r_{m}$. The next step is to evaluate the current cuckoo. For that, we apply the measure operation in order to get a binary solution which represents a potential solution. The binary solution is evaluated by using the number of used bins as objective function. After this step, we apply the interference operation according to the best current element. We replace some worst nests by the current cuckoo if it is better or by new random nests generated by the Lévy flight. The selection phase in QICSA of the best nests or solutions is comparable to some form of elitism selection used in genetic algorithms, which ensures the best solution is kept always in the next iteration. Finally, the global best solution is then updated if a better one is found and the whole process is repeated until reaching a stopping criterion. In more details, the proposed QICSA can be described as in figure 10 .

The particularity of QICSA algorithm stems from the quantum representation it adopts which allows representing the superposition of all potential solutions for a given problem. Moreover, the position of a nest depends on the probability amplitudes $a$ and $b$ of the qubit function. The probabilistic nature of the quantum measure offers a good diversity to the cuckoo search algorithm, while the interference operation helps to intensify the search around the good solutions.

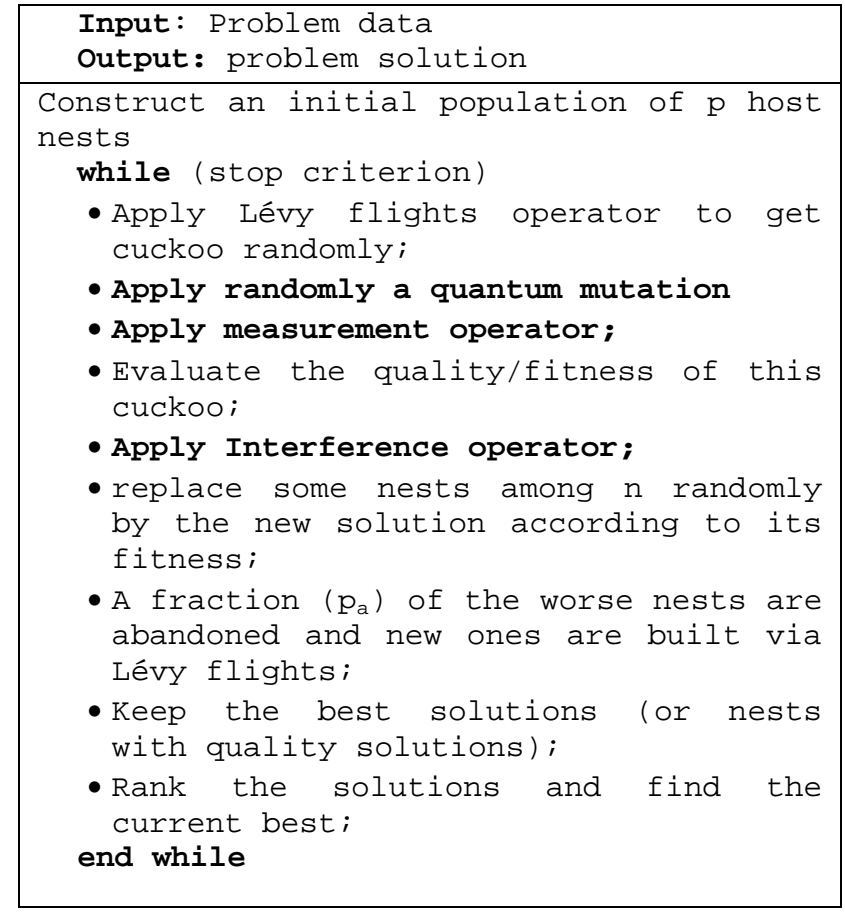

Fig. 10. Quantum Inspired Cuckoo Search Schema.

\section{Implementation and Validation}

We have implemented our approach in Matlab 7.7 and tested on home PC with core duo processor and 2.2 GB of memory. In order to evaluate the proposed approach, we have used a set of benchmark data sets taken from the site http://www.wiwi.unijena.de/Entscheidung/binpp/. The benchmark data sets are divided into three classes: easy, medium and hard class instances. In all experiment, we have used 5000 iterations and 6 nests in the population.

The obtained results are summarized in Table 1., Table 2., and Table 3., the first column is the name of the instance, the second column contains the number of variables, the third contains the bin capacity, the fourth column contains the results of the first fit decreasing heuristic (FFD), the fifth column contains the results our approach (QICSABP), and the last column contains the best know results. Moreover, we have used the Friedman tests for comparing statistically the found solutions.

In term of rapidity, the proposed algorithm is slower than the FFD algorithm. However, in terms of solutions quality, the QICSABP algorithm is better than the FFD in some instances of medium and hard classes of tests (Table 3, table 4) as it's showed by the Freidman tests (Figures 11, 12). Table 1 shows the results of the first series of easy-type instances, the obtained results of our algorithm and those of FFD are completely identical to the best known solutions. In the second series of medium instances, our algorithm is better than the FFD heuristic in 5 instances, while this later is better than our algorithm in only one test. In the Freidman test, our approach is the closest to the best know solutions compared to the FFD algorithm (Figure 
11). Indeed, our algorithm has found 4 best solutions (table). Finally, Table 3 shows the results for the third series of hard instances. Our algorithm ranks second in the Friedman test after the best known solutions (figure 12). In this class, our algorithm is better than the FFD in three instances. Moreover, the differences between our results and the best known results are between 2 and 4.

The effectiveness of our approach is explained by the good combination between the diversification of the random hybrid measure operation and the intensification of the interference operation which leads the algorithm to explore effectively the search space and locate a good solution.

Table 2: The bin packing results for the easy class, n: number of items, C: bin capacity.

\begin{tabular}{|l|l|l|l|c|c|}
\hline Instance & $\boldsymbol{N}$ & Cap & FFD & QICSABP & $\begin{array}{l}\text { Best } \\
\text { Known }\end{array}$ \\
\hline N1C1W1_A & 50 & 100 & 25 & 25 & 25 \\
\hline N1C1W1_D & 50 & 100 & 28 & 28 & 28 \\
\hline N1C1W1_G & 50 & 100 & 25 & 25 & 25 \\
\hline N1C1W1_B & 50 & 100 & 31 & 31 & 31 \\
\hline N1C1W1_E & 50 & 100 & 26 & 26 & 26 \\
\hline N1C1W1_F & 50 & 100 & 27 & 27 & 27 \\
\hline N1C1W1_I & 50 & 100 & 25 & 25 & 25 \\
\hline N2C1W2_P & 100 & 100 & 68 & 68 & 68 \\
\hline N2C1W2_N & 100 & 100 & 64 & 64 & 64 \\
\hline N2C1W2_O & 100 & 100 & 64 & 64 & 64 \\
\hline N2C1W2_R & 100 & 100 & 67 & 67 & 67 \\
\hline N4C1W2_T & 500 & 100 & 323 & 323 & 323 \\
\hline N4C1W4_C & 500 & 100 & 365 & 365 & 365 \\
\hline N4C1W4_A & 500 & 100 & 368 & 368 & 368 \\
\hline N4C1W4_D & 500 & 100 & 359 & 359 & 359 \\
\hline N4C1W4_B & 500 & 100 & 349 & 349 & 349 \\
\hline
\end{tabular}

Table 3: The bin packing results for the medium class, $n$ : number of items, C: bin capacity.

\begin{tabular}{|l|c|c|c|c|c|}
\hline Instance & $\boldsymbol{N}$ & Cap & $\boldsymbol{F F}$ & QICSABP & $\begin{array}{l}\text { Best } \\
\text { Known }\end{array}$ \\
\hline N1W1B2R1 & 50 & 1000 & 18 & 17 & 17 \\
\hline N1W1B1R9 & 50 & 1000 & 19 & 18 & 17 \\
\hline N1W1B1R2 & 50 & 1000 & 18 & 18 & 17 \\
\hline N1W1B2R0 & 50 & 1000 & 18 & 18 & 17 \\
\hline N1W1B2R3 & 50 & 1000 & 17 & 17 & 16 \\
\hline N2W1B1R0 & 100 & 1000 & 37 & 36 & 34 \\
\hline N2W1B1R3 & 100 & 1000 & 38 & 37 & 34 \\
\hline N2W1B1R1 & 100 & 1000 & 37 & 37 & 34 \\
\hline N2W1B1R4 & 100 & 1000 & 37 & 37 & 34 \\
\hline N2W3B3R7 & 100 & 1000 & 13 & 13 & 13 \\
\hline N2W4B1R0 & 100 & 1000 & 12 & 12 & 12 \\
\hline N4W2B1R0 & 500 & 1000 & 109 & 109 & 101 \\
\hline N4W2B1R3 & 500 & 1000 & 109 & 108 & 100 \\
\hline N4W3B3R7 & 500 & 1000 & 74 & 74 & 74 \\
\hline N4W4B1R0 & 500 & 1000 & 58 & 58 & 56 \\
\hline N4W4B1R1 & 500 & 1000 & 58 & 58 & 56 \\
\hline
\end{tabular}

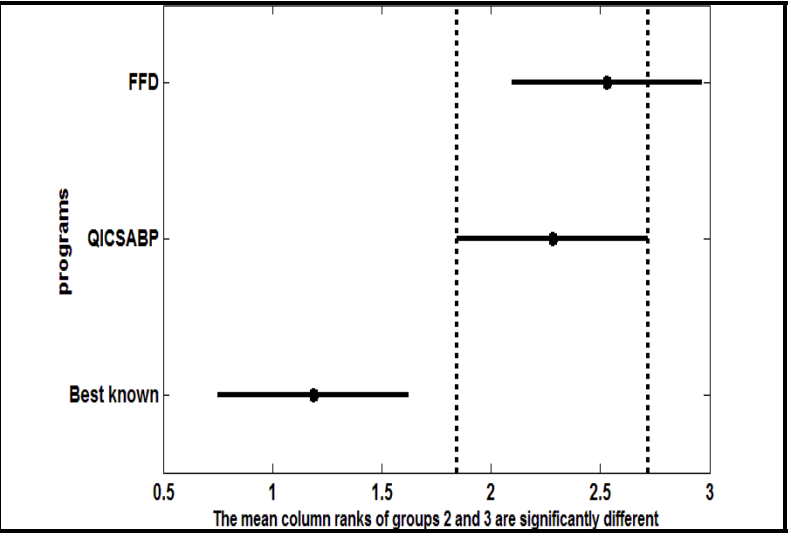

Fig. 11. Friedman test for medium-type instances.

Table 4: The bin packing results for the Hard class, n: number of items, C: bin capacity.

\begin{tabular}{|l|l|l|l|l|l|}
\hline Instance & $\boldsymbol{N}$ & Cap & FFD & QCSBPP & $\begin{array}{l}\text { Best } \\
\text { Known }\end{array}$ \\
\hline HARD0 & 200 & 100000 & 59 & 59 & 56 \\
\hline HARD1 & 200 & 100000 & 60 & 60 & 57 \\
\hline HARD2 & 200 & 100000 & 60 & 60 & 56 \\
\hline HARD3 & 200 & 100000 & 59 & 59 & 55 \\
\hline HARD4 & 200 & 100000 & 60 & 60 & 57 \\
\hline HARD5 & 200 & 100000 & 59 & 59 & 56 \\
\hline HARD6 & 200 & 100000 & 60 & 59 & 57 \\
\hline HARD7 & 200 & 100000 & 59 & 59 & 55 \\
\hline HARD8 & 200 & 100000 & 60 & 59 & 57 \\
\hline HARD9 & 200 & 100000 & 60 & 59 & 56 \\
\hline
\end{tabular}

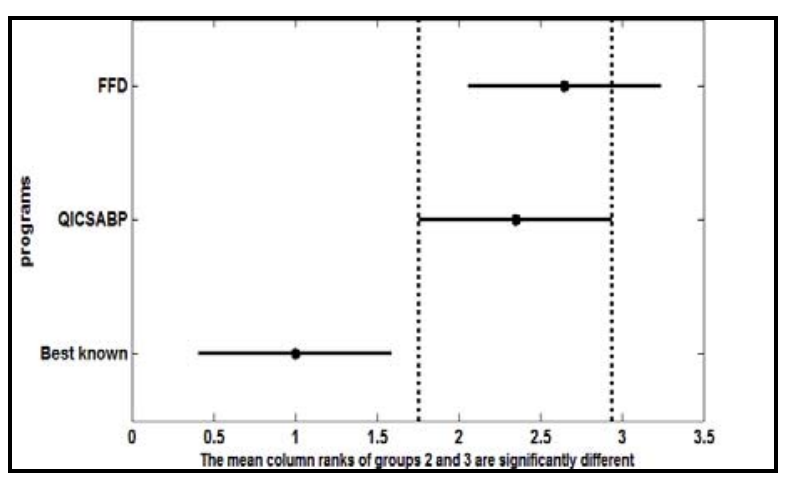

Fig.12. Friedman test for HARD-type instances.

\section{Conclusion}

We have tackled in this paper the one-dimensional bin packing problem. The importance of this problem lies in its widespread applications in transport, loading and industry. Unfortunately, this problem belongs to the class of NP-hard problems. To solve this problem, we have proposed in this paper, a new stochastic method based on the quantum inspired cuckoo search algorithm. The main contributions of our approach are the use a new hybrid measure operation which integrates the FF heuristic in the core of the standard measure operation. The FF is applied if the measure operation doesn't succeed to pack an item in a bin. The great feature of 
this hybrid measure is its great ability to generate different good random solutions. The proposed algorithm reduces efficiently the population size and the number of iterations to have the optimal solution, thanks to quantum representation, solutions allows the coding of all the potential solutions with a certain probability. Moreover, the interference allows to intensify the search is around the best solutions. The quantum levy flight helps also to apply a random walk around the best solution. The results are very encouraging and clearly show the effectiveness of our approach. As perspective, we want to test the effectiveness of the use of local search methods such as tabu search, variable neighbourhood search, etc. we can also use other heuristics to build the initial solution like FFD or BFD.

\section{References}

[1] Martello, S., and Toth, P. Bin-packing problem. In Knapsack Problems: Algorithms and Computer Implementations.Wiley, 1990, (8): 221-245.

[2] Coffman, E. G., Jr., Garey, M. R., and Johnson, D. S. Approximation algorithms for bin packing: A survery. In D. Hochbaum, editor, Appoximation algorithms for NP-Hard Problems, 1996, 46-93. PWS Publishing, Boston.

[3] Fleszar, K., Hindi, K. S., 2002. New heuristics for one-dimensional bin-packing. Computers and Operations Research, 29 (7): 821-839.

[4] lvim, A.C.F., Ribeiro, C.C., Glover, F., Aloise, D.J. A Hybrid Improvement Heuristic for the OneDimensional Bin Packing Problem. Journal of Heuristics, 2004, (10):205-229.

[5] Kao, C.-Y. and F.-T. Lin. A stochastic approach for the one-dimensional bin-packing problems. In Systems, Man and Cybernetics, 1992, (2): 15451551.

[6] Scholl, A., R. Klein, and C. Juergens (1997). Bison: A fast hybrid procedure for exactly solving the one-dimensional bin packing problem. Computers \& Operations Research,1997, 24(7): 627-645.

[7] Falkenauer, E. A hybrid grouping genetic algorithm for bin packing. Journal of Heuristics, 1996, (2):530 .

[8] Wang, S., Shi, R., Wang, L. and Ge, M. Study on improved ant colony optimization for bin-packing problem, International Conference on Computer Desingn and Application, 2010,(4):489-491 .

[9] Jourdan, L., Basseur, M. and Talbi, E.G., Hybridizing exact methods and metaheuristics: a taxonomy. European Journal of Operational Research. Vol. 199. 620-629, 2009.

[10] Fogel, D.B., An Introduction to Evolutionary Computation, Tutorial, Congress on Evolutionary Computation (CEC'2001), Seoul, Korea, 2001.

[11] Dorigo M. and Gambardella, L.M. Ant Colony System : A Cooperative Learning Approach to the Traveling Salesman Problem, IEEE Transactions on Evolutionary Computation, Vol.1, N. 1, pp. 5366, 1997.

[12] Hu, X, Zhang, J. and Li, Y. Orthogonal methods based ant colony search for solving continuous optimization problems. Journal of Computer Science and Technology, 23(1), pp.2-18, 2008.

[13] Nabti, S. and Meshoul, S. Predator Prey Optimisation for Snake Based Contour Detection, International Journal of Intelligent Computing and Cybernetics - IJICC, Emerald Publishers, 2(2), pp.228-242, 2009.

[14] Clerc, M., and Kennedy, J. The particle swarm explosion, stability, and convergence in a multidimensional complex space. IEEE Trans. Evo. Comp., 6(1) 58-73, 2002.

[15] Eberhart, R. C., Shi, Y. and Kennedy, J: Swarm Intelligence. The Morgan Kaufmann Series in Artificial Intelligence. Morgan Kaufmann, San Francisco, CA, USA, 2001.

[16] Yang, X.-S., and Deb, S., Engineering Optimisation by Cuckoo Search, Int. J. Mathematical Modelling and Numerical Optimisation, Vol. 1, No. 4, 330-343, 2010.

[17] Barthelemy, P., Bertolotti, J., Wiersma, D. S., 2008. A Lévy flight for light. Nature, 453, 495-498.

[18] Payne, R. B., Sorenson, M. D., and Klitz, K. The Cuckoos, Oxford University Press, 2005.

[19] Pavlyukevich, I. Lévy flights, non-local search and simulated annealing, J. Computational Physics, 226, 1830-1844, 2007.

[20] Tein L. H. and Ramli R., Recent advancements of nurse scheduling models and a potential path, in: Proc. 6th IMT-GT Conference on Mathematics, Statistics and its Applications (ICMSA 2010), pp. 395-409, 2010.

[21] Dhivya, M. Energy Efficient Computation of Data Fusion in Wireless Sensor Networks Using Cuckoo Based Particle Approach (CBPA), Int. J. of Communications, Network and System Sciences, Vol. 4, No. 4, 249-255, 2011.

[22] A. Gherboudj, A. Layeb, S. Chikhi.: Solving 0-1 knapsack problems by a discrete binary version of cuckoo search algorithm. to appear in the International Journal of Bio-Inspired Computation, ISSN 1758-0366, Inderscience publisher, 2012

[23] Jaeger, G. Quantum Information: An Overview. Berlin: Springer. 2006

[24] Han, K.H. and Kim, J.H.. "Quantum-inspired Evolutionary Algorithms with a New Termination Criterion, He Gate, and Two Phase Scheme," IEEE Transactions on Evolutionary Computation, IEEE Press, vol. 8, no. 2, pp. 156-169, April 2004.

[25] Layeb, A., Saidouni, D.E. A New Quantum Evolutionary Local Search Algorithm for MAX 3SAT Problem. In Proceedings of the 3rd international Workshop on Hybrid Artificial intelligence Systems. Lecture Notes in Artificial Intelligence, vol. 5271. Springer-Verlag, Berlin, Heidelberg, (2008)172-179. 
[26] Draa A., Meshoul S., Talbi H., Batouche A Quantum-Inspired Differential Evolution Algorithm for Solving the N-Queens Problem. Int. Arab J. Inf. Technol. 7(1):pp. 21-27, 2010.

[27] Layeb, A. Hybrid Quantum Scatter Search Algorithm for Combinatorial Optimization Problems. In the journal of Annals. Computer Science Series, ISSN: 1583-7165, Vol. 8, No.2, pp.227-244, 2010

[28] Layeb, A., Saidouni, D.E. A New Quantum Evolutionary Algorithm with Sifting Strategy for Binary Decision Diagram Ordering Problem, in the International Journal of Cognitive Informatics and Natural Intelligence. ISSN 1557-3958, Vol. 4. No 4, pp. 47-61, December 2010.

[29] Layeb: A novel quantum inspired cuckoo search for knapsack problems, in the International Journal of Bio-Inspired Computation, ISSN 1758-0366, Vol. 3, No. 5, pp.297-305, Inderscience publisher, 2011.

Abdesslem Layeb is Senior Lecturer in the department of computer science at the University of Constantine. He received his $\mathrm{PhD}$ degree in computer science from the University Mentouri of Constantine, Algeria. Dr. Layeb is interested to the combinatorial optimization methods and its application to solve several problems from different domains like Bioinformatics, imagery, formal methods. Dr. Layeb has many publications in theoretical computer science, bioinformatics, and formal methods.

Seriel Rayene Boussalia is Master research student from Mentouri University of Constantine. Her main interests are the bin packing problems and the combinatorial optimization methods. 